\title{
SINGLE PHYSICAL PERFORMANCE MEASURES CANNOT IDENTIFY GERIATRIC OUTPATIENTS WITH SARCOPENIA
}

\author{
S.M.L.M. LOOIJAARD ${ }^{1}$, S.J. OUDBIER ${ }^{1}$, E.M. REIJNIERSE ${ }^{2}$, G.J. BLAUW ${ }^{3,4}$, \\ C.G.M. MESKERS ${ }^{5}$, A.B. MAIER ${ }^{2,6}$
}

\begin{abstract}
1. Department of Internal Medicine, Section of Gerontology and Geriatrics, VU University Medical Center, Boelelaan 1117, 1081 HV, Amsterdam, The Netherlands; 2. Department of

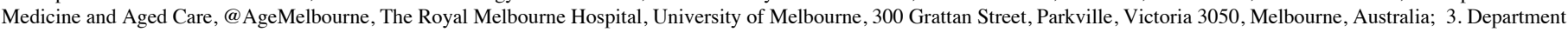
of Gerontology and Geriatrics, Leiden University Medical Center, Albinusdreef 2, 2333 ZA, Leiden, The Netherlands; 4. Department of Geriatrics, Bronovo Hospital, Bronovolaan 5, 2597 AX, The Hague, The Netherlands; 5. Department of Rehabilitation Medicine, VU University Medical Center, Boelelaan 1117, 1081 HV, Amsterdam, The Netherlands;

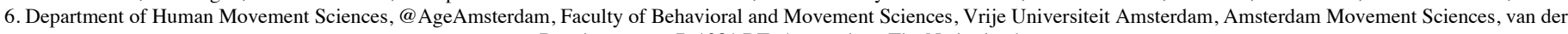
Boechorststraat 7, 1081 BT, Amsterdam, The Netherlands.

Corresponding author: A.B. Maier, Department of Human Movement Sciences, @Age, Faculty of Behavioral and Movement Sciences, Vrije Universiteit Amsterdam, Amsterdam Movement Sciences, van der Boechorststraat 7, 1081 BT, Amsterdam, The Netherlands, Telephone number: 020-5982000 a.b.maier@vu.nl
\end{abstract}

\begin{abstract}
Background: Sarcopenia is highly prevalent in the older population and is associated with several adverse health outcomes. Equipment to measure muscle mass and muscle strength to diagnose sarcopenia is often unavailable in clinical practice due to the related expenses while an easy physical performance measure to identify individuals who could potentially have sarcopenia is lacking. Objectives: This study aimed to assess the association between physical performance measures and definitions of sarcopenia in a clinically relevant population of geriatric outpatients. Design, setting and participants: A cross-sectional study was conducted, consisting of 140 community-dwelling older adults that were referred to a geriatric outpatient clinic. No exclusion criteria were applied. Measurements: Physical performance measures included balance tests (sideby-side, semi-tandem and tandem test with eyes open and -closed), four-meter walk test, timed up and go test, chair stand test, handgrip strength and two subjective questions on mobility. Direct segmental multi-frequency bioelectrical impedance analysis was used to measure muscle mass. Five commonly used definitions of sarcopenia were applied. Diagnostic accuracy was determined by sensitivity, specificity and area under the curve. Results: Physical performance measures, i.e. side-by-side test, tandem test, chair stand test and handgrip strength, were associated with at least one definition of sarcopenia. Diagnostic accuracy of these physical performance measures was poor. Conclusions: Single physical performance measures could not identify older individuals with sarcopenia, according to five different definitions of sarcopenia.
\end{abstract}

Key words: Aged, geriatrics, physical performance, sarcopenia.

\section{Introduction}

Prevalence rates of sarcopenia, defined as age-related low muscle mass and muscle strength, vary between $0 \%$ and $15 \%$ in healthy older individuals and between $2 \%$ and $34 \%$ in geriatric outpatients, depending on the applied definition (1). Sarcopenia is associated with decreased mobility, a higher risk of falls, dependency in activities of daily living, morbidity and mortality (2-4). Although there is no consensus yet on the definition of sarcopenia, the majority of definitions contain a measure of muscle mass and/or muscle strength (5-10).

Equipment to measure muscle mass and muscle strength is often unavailable in clinical practice due to the related expenses (11). Poor physical performance has been shown to be associated with sarcopenia (12-14). Recently, a prediction model was proposed to identify sarcopenia with the use of demographic parameters and physical performance measures (15). However, such a prediction model is time consuming due to its inclusion of multiple physical tests and complex calculations. Identifying individuals who could potentially have sarcopenia in clinical practice would be greatly facilitated Received April 1, 2018 when an easy to use physical performance measure could be applied to identify individuals with sarcopenia. This could lead to the identification of individuals who could potentially have sarcopenia, who could then be referred to diagnose sarcopenia.

This study aims to assess the association between physical performance measures and different definitions of sarcopenia in a clinically relevant population of geriatric outpatients.

\section{Methods}

\section{Study design}

This cross-sectional study consisted of 140 communitydwelling older adults who were referred to a geriatric outpatient clinic of a middle-sized teaching hospital (Bronovo, The Hague, The Netherlands) between March 2011 and January 2012. These older adults were referred because of mobility problems for a comprehensive geriatric assessment. The study originally consists of 299 older adults, but muscle mass measurements were only available in 140 older adults as these measurements were later added as part of clinical care. No exclusion criteria were applied; inclusion was based 


\section{THE JOURNAL OF FRAILTY \& AGING}

on referral. The study was approved by the Medical Ethical Committee of the Leiden University Medical Center (Leiden, the Netherlands). Informed consent was waived as this study was based on regular care.

\section{Study population characteristics}

Medical charts were used to retrieve information on age, sex and medical history. Medical history included information on the presence of: hypertension, myocardial infarction, chronic obstructive pulmonary disease, diabetes mellitus, rheumatoidor osteoarthritis, Parkinson's disease and malignancy. The presence of two or more of these diseases was defined as multimorbidity. Anthropometric measurements included height and body weight and were measured to the nearest 0.1 decimal. Cognitive functioning was measured by the Mini Mental State Examination (MMSE) resulting in a score ranging from 0 to 30 points, higher scores indicating better cognitive function (16).

\section{Physical performance measures, objective}

Physical performance measures included balance tests, fourmeter walk test, timed up and go (TUG), chair stand test (CST) and handgrip strength (HGS).

Balance tests were performed in three different positions (side-by-side, semi-tandem and tandem) according to the protocol of the Short Physical Performance Battery (17), and were performed with eyes open and with eyes closed. Individuals were classified as unable to maintain for ten seconds (0) and able to maintain for ten seconds (1). Tandem balance test with eyes closed was excluded from the present analysis as the number of individuals who were able to maintain in the tandem position for ten seconds was less than five individuals.

Gait speed was obtained by a four-meter walk test where individuals were asked to walk at their usual pace (17). The best performance of two measurements was used and expressed in meters per second.

The TUG measures the time in seconds needed to stand up from a sitting position without using hands, walk three meters, walk around a cone, walk three meters back and return to sitting position without using hands, as fast as possible.

The CST measures the time in seconds needed to stand up five times from sitting position to a straight standing position and sit down again while keeping the arms crossed over the chest, as fast as possible (17).

HGS was measured using a hydraulic handheld dynamometer (Jamar, Sammons Preston, Inc., Bolingbrook, IL, USA). Individuals were asked to squeeze as hard as possible three times with the right and left hand side alternately. Maximal HGS of the three trials was used for analysis (18) and expressed in kilograms.

Higher gait speed and HGS implied a better physical performance while a higher TUG and CST time implied a lower physical performance. For all physical performance measures, all individuals who could not perform or finish the test or used hands to stand up from a sitting position, were given a time of 100 seconds.

\section{Physical performance measures, subjective}

In addition to the objective physical performance measures, two questions were asked: 1) Falls: "Did you fall in the past year?" (yes/no) and 2) Difficulty with walking: "Do you experience difficulty with walking?" (yes/no).

\section{Sarcopenia definitions}

Muscle mass was measured using direct segmental multifrequency Bioelectrical Impedance Analysis (DSM-BIA; InBody 720, Biospace Co., Ltd, Seoul, Korea) (19). Five definitions of sarcopenia were used to examine the association between physical measures and sarcopenia: 1) Baumgartner et al. using appendicular lean mass (ALM) divided by height2 (5); 2) Janssen et al. using relative skeletal muscle mass (SM) (SM divided by body mass) (6); 3) European Working Group on Sarcopenia in Older Persons (EWGSOP) using an algorithm of gait speed, HGS and skeletal muscle index (SMI; SM divided by height2) (7); 4) Foundation for National Institutes of Health (FNIH) definition one using HGS and ALM divided by body mass index (BMI) (8) and 5) The International Working Group on Sarcopenia (IWGS) using gait speed and ALM divided by height2 (9).

\section{Statistical analysis}

Continuous variables were reported by mean \pm standard deviation (SD) if data was normally distributed or median [interquartile range (IQR)] for skewed distributions. Associations between physical performance measures (independent variables) with definitions of sarcopenia (dependent variables) were analyzed with binary logistic regression analysis. Two models were used: the crude model and an adjusted model for sex and age. P-values of less than 0.05 were considered statistically significant.

For the statistically significant associations between physical performance measures and definitions of sarcopenia, sensitivity, specificity and the area under the curve (AUC) were calculated to determine the diagnostic accuracy. Sensitivity and specificity were defined as low $<70 \%$, moderate $70-90 \%$ and high $>90 \%$. AUC was defined as low $<0.70$, acceptable $0.70-0.80$, excellent $0.80-0.90$ and outstanding $>0.90$. To test diagnostic accuracy, CST and HGS were dichotomized: CST $\geq 13$ seconds (14, 20 ), and HGS $<20$ kilograms for females and $<30$ kilograms for males were considered low (21). Statistical analyses were performed using Statistical Package for Social Sciences (SPSS Inc, Chicago, USA), version 22.

\section{Results}

Table 1 shows the characteristics of the geriatric outpatients, with a mean age of 80.9 (7.1) years and $42 \%$ males. Table 2 shows the applied definitions of sarcopenia and the prevalence 


\section{PHYSICAL PERFORMANCE AND SARCOPENIA}

of sarcopenia. Prevalence of sarcopenia ranged from $3.6 \%$ to $23.6 \%$, depending on the definition.

\section{Table 1}

Characteristics of geriatric outpatients $(\mathrm{N}=140)$

\begin{tabular}{|c|c|c|}
\hline & $\mathbf{N}$ & Total \\
\hline \multicolumn{3}{|l|}{ General characteristics } \\
\hline Sex, male & 140 & $59(42.1)$ \\
\hline Age, years, mean \pm SD & 140 & $80.9 \pm 7.08$ \\
\hline Body weight, $\mathrm{kg}$, mean $\pm \mathrm{SD}$ & 140 & $71.9 \pm 15.2$ \\
\hline Height, $\mathrm{cm}$, mean $\pm \mathrm{SD}$ & 140 & $167.2 \pm 9.93$ \\
\hline \multicolumn{3}{|l|}{ Health characteristics } \\
\hline Multimorbidity & 134 & $55(41.0)$ \\
\hline MMSE, median [IQR] & 140 & 27 [25-29] \\
\hline \multicolumn{3}{|l|}{ Physical performance measures, objective } \\
\hline Side-by-side eyes open, able to maintain & 140 & $135(96.4)$ \\
\hline Semi-tandem eyes open, able to maintain & 140 & $120(85.7)$ \\
\hline Tandem eyes open, able to maintain & 140 & $53(37.9)$ \\
\hline Side-by-side eyes closed, able to maintain & 138 & $114(82.6)$ \\
\hline Semi-tandem eyes closed, able to maintain & 138 & $69(50.0)$ \\
\hline Tandem eyes closed, able to maintain & 138 & $3(2.2)$ \\
\hline Gait speed, $\mathrm{m} / \mathrm{s}$, mean $\pm \mathrm{SD}$ & 140 & $0.77 \pm 0.27$ \\
\hline TUG, sec, median [IQR] & 135 & $16.6[12.1-26.2]$ \\
\hline CST, sec, median [IQR] & 138 & $15.6[11.9-26.5]$ \\
\hline $\mathrm{HGS}, \mathrm{kg}$, mean $\pm \mathrm{SD}$, males & 59 & $33.1 \pm 6.01$ \\
\hline $\mathrm{HGS}, \mathrm{kg}$, mean $\pm \mathrm{SD}$, females & 81 & $21.3 \pm 5.11$ \\
\hline \multicolumn{3}{|l|}{ Physical performance measures, subjective } \\
\hline History of falling last 12 months, yes & 140 & $89(63.6)$ \\
\hline Difficulty walking last 12 months, yes & 140 & $101(72.1)$ \\
\hline \multicolumn{3}{|l|}{ Muscle mass parameters } \\
\hline $\mathrm{ALM} / \mathrm{height} 2, \mathrm{~kg} / \mathrm{m} 2$, mean $\pm \mathrm{SD}$ & 140 & $7.16 \pm 1.21$ \\
\hline $\mathrm{ALM} / \mathrm{BMI}$, mean $\pm \mathrm{SD}$ & 140 & $0.80 \pm 0.21$ \\
\hline $\mathrm{SMI}, \mathrm{kg} / \mathrm{m} 2$, mean $\pm \mathrm{SD}$ & 140 & $9.21 \pm 1.31$ \\
\hline $\mathrm{SM} /$ body mass, $\%$, mean $\pm \mathrm{SD}$ & 140 & $64.0 \pm 8.79$ \\
\hline $\begin{array}{l}\text { All results are given in number (percentage) un } \\
\text { deviation; MMSE: Mini Mental State Examinatio } \\
\text { TUG: Timed Up and Go; CST: Chair Stand T } \\
\text { Appendicular Lean Mass; BMI: Body Mass Ind } \\
\text { Skeletal Muscle. }\end{array}$ & ted & $\begin{array}{l}\text { vise. SD: standard } \\
\text { nterquartile range; } \\
\text { Strength; ALM: } \\
\text { Guscle Index; SM: }\end{array}$ \\
\hline
\end{tabular}

Table 3 shows the association between physical performance measures and sarcopenia according to the applied definitions. Out of all balance tests, the ability to perform the tandem stance with eyes open was most often associated with a decreased likelihood to have sarcopenia (Baumgartner et al., EWGSOP and IWGS). CST was associated with sarcopenia by use of the EWGSOP and IWGS definitions. HGS was associated with sarcopenia using the definition of Baumgartner et al. and IWGS. The other physical performance measures i.e. semi- tandem balance test with eyes open and eyes closed, gait speed, TUG and the subjective physical performance measures did not show an association with any of the definitions of sarcopenia.

Table 4 shows the diagnostic accuracy for physical performance measures significantly associated with sarcopenia. The tandem balance test with eyes open showed moderate sensitivity and low specificity and AUC for all three sarcopenia definitions. The side-by-side test with eyes closed showed low sensitivity, moderate specificity and low AUC for the EWGSOP definition. CST showed low sensitivity, specificity and AUC for the EWGSOP and IWGS definitions. HGS showed low sensitivity, moderate specificity and low AUC for the Baumgartner et al. and IWGS definitions.

\section{Discussion}

Physical performance measures, i.e. side-by-side test, tandem test, CST and HGS, were associated with sarcopenia using several definitions, but diagnostic accuracy was poor.

Previous studies have shown that tandem balance test, gait speed, CST and HGS are valid and reliable measures to assess physical performance $(22,23)$. Moreover, gait speed, CST and HGS have proven to be associated with sarcopenia according to the EWGSOP definition in community dwelling older adults $(20,24,25)$. Unfortunately, the results of this study in geriatric outpatients did not show a single suitable physical performance measure to identify older individuals with sarcopenia. For a test to be suitable to identify individuals with a high risk on sarcopenia, it needs to have a high sensitivity as especially false-negatives are undesirable. Sensitivity was low to moderate, which would mean that many individuals who could potentially have sarcopenia would be missed. Moreover, specificity and AUC were low and therefore single physical performance measures have poor diagnostic accuracy to identify individuals with sarcopenia.

Sarcopenia is associated with negative health outcomes and therefore an important diagnosis in clinical practice. Nutritional and physical interventions have proven to increase muscle mass, muscle strength and physical performance $(26,27)$. Improving physical performance measures might reduce the risk of sarcopenia and therewith its negative health outcomes. Early recognition of sarcopenia is necessary to initiate interventions. BMI is a measurement that is often used to identify individuals who are at risk of adverse health outcomes, however, BMI does not encompass the risk of sarcopenia (28) Another proposed screening tool to identify individuals with sarcopenia is the SARC-F, a simple five-item questionnaire (subjective measures of strength, assistance in walking, rise from a chair, climb stairs, falls). This is one of the screening instruments that is increasingly being used in communitydwelling middle-aged to older adults to identify individuals who could potentially have sarcopenia $(29,30)$.

Multimorbidity was high in this population of geriatric outpatients and this could be an explanation for the lack of 


\section{THE JOURNAL OF FRAILTY \& AGING}

Table 2

Prevalence of sarcopenia in geriatric outpatients according to the applied definitions of sarcopenia

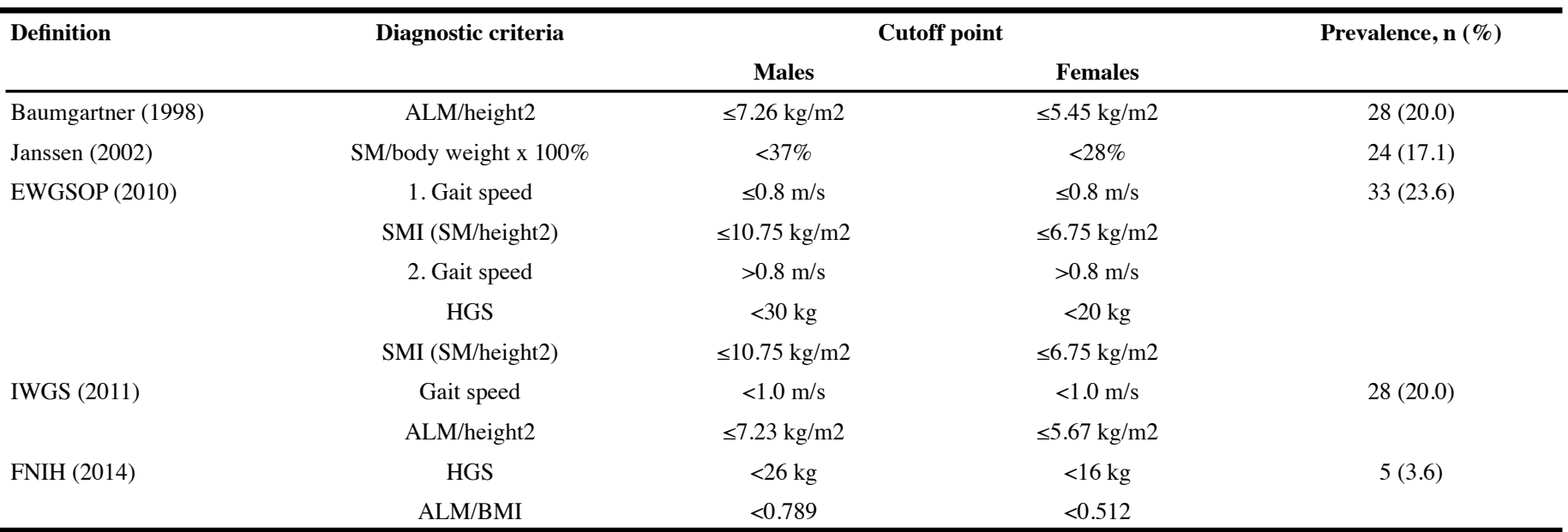

ALM: Appendicular Lean Mass; SM: Skeletal Muscle; EWGSOP: European Working Group on Sarcopenia in Older People; HGS: Handgrip Strength; SMI: Skeletal Muscle Index; IWGS: International Working Group on Sarcopenia; FNIH: Foundation for the National Institutes of Health; BMI: Body Mass Index.

Table 3

Physical performance measures and sarcopenia according to the applied definitions

\begin{tabular}{|c|c|c|c|c|c|c|c|c|c|c|}
\hline & \multicolumn{2}{|c|}{ Baumgartner } & \multicolumn{2}{|c|}{ Janssen } & \multicolumn{2}{|c|}{ EWGSOP } & \multicolumn{2}{|c|}{ IWGS } & \multicolumn{2}{|c|}{ FNIH } \\
\hline & OR & $95 \%$ CI & OR & $95 \% \mathrm{CI}$ & OR & $95 \% \mathrm{CI}$ & OR & $95 \% \mathrm{CI}$ & OR & $95 \% \mathrm{CI}$ \\
\hline Side-by-side, eyes open* & 1.00 & $0.11-9.31$ & 0.82 & $0.09-7.69$ & 1.24 & $0.13-11.5$ & 0.36 & $0.06-2.25$ & 0.12 & $0.01-1.36$ \\
\hline Semi-tandem, eyes open* & 1.49 & $0.41-5.49$ & 0.80 & $0.24-2.65$ & 0.51 & $0.19-1.42$ & 1.00 & $0.31-3.27$ & 0.66 & $0.07-6.18$ \\
\hline Adjusted model & 1.74 & $0.46-6.65$ & 0.61 & $0.17-2.18$ & 0.25 & $0.06-1.02$ & 1.25 & $0.37-4.26$ & 0.45 & $0.04-4.70$ \\
\hline Tandem, eyes open* & 0.29 & $0.10-0.82$ & 1.83 & $0.75-4.44$ & 0.44 & $0.18-1.07$ & 0.29 & $0.10-0.82$ & 1.10 & $0.18-6.80$ \\
\hline Side-by-side, eyes closed* & 0.52 & $0.19-1.41$ & 0.76 & $0.25-2.29$ & 0.23 & $0.09-0.57$ & 0.43 & $0.16-1.13$ & 0.84 & $0.09-7.83$ \\
\hline Adjusted model & 0.58 & $0.21-1.65$ & 0.66 & $0.21-2.10$ & 0.07 & $0.01-0.33$ & 0.51 & $0.18-1.40$ & 0.66 & $0.07-6.58$ \\
\hline Semi-tandem, eyes closed* & 0.76 & $0.33-1.77$ & 0.82 & $0.34-1.98$ & 0.67 & $0.30-1.48$ & 0.48 & $0.20-1.13$ & 1.52 & $0.25-9.41$ \\
\hline Adjusted model & 0.94 & $0.38-2.34$ & 0.62 & $0.23-1.66$ & 0.51 & $0.19-1.39$ & 0.63 & $0.25-1.59$ & 1.09 & $0.16-7.74$ \\
\hline Gait speed, $\mathrm{m} / \mathrm{s}$ & 0.41 & $0.08-2.07$ & 1.54 & $0.30-7.83$ & NA & NA & NA & NA & 0.72 & $0.02-21.4$ \\
\hline CST, sec & 1.01 & $1.00-1.02$ & 1.00 & $0.99-1.01$ & 1.01 & $0.99-1.02$ & 1.01 & $1.00-1.02$ & 1.01 & $0.99-1.03$ \\
\hline Adjusted model & 1.01 & $1.00-1.02$ & 1.00 & $0.99-1.08$ & 1.02 & $1.00-1.03$ & 1.01 & $1.00-1.02$ & 1.02 & $0.99-1.04$ \\
\hline $\mathrm{HGS}, \mathrm{kg}$ & 0.97 & $0.92-1.02$ & 1.03 & $0.98-1.09$ & NA & NA & 0.96 & $0.91-1.01$ & NA & NA \\
\hline Adjusted model & 0.86 & $0.78-0.94$ & 0.94 & $0.87-1.03$ & NA & NA & 0.85 & $0.77-0.94$ & NA & NA \\
\hline Difficulty walking, yes & 0.77 & $0.32-1.89$ & 2.16 & $0.69-6.79$ & 1.04 & $0.43-2.49$ & 1.20 & $0.47-3.10$ & 1.57 & $0.17-14.5$ \\
\hline Adjusted model & 0.68 & $0.27-1.71$ & 2.34 & $074-7.44$ & 1.96 & $0.70-5.47$ & 1.16 & $0.43-3.15$ & 1.72 & $0.18-16.0$ \\
\hline History of falling, yes & 1.27 & $0.53-3.06$ & 0.95 & $0.38-2.35$ & 1.00 & $0.45-2.26$ & 1.56 & $0.63-3.85$ & 2.35 & $0.26-21.6$ \\
\hline Adjusted model & 1.25 & $0.51-3.04$ & 0.94 & $0.38-2.33$ & 1.29 & $0.50-3.32$ & 1.61 & $0.63-4.09$ & 2.25 & $0.24-21.0$ \\
\hline
\end{tabular}

OR: Odds Ratio; CI: Confidence interval; EWGSOP: European Working Group on Sarcopenia in Older People; IWGS: International Working Group on Sarcopenia; FNIH: Foundation for

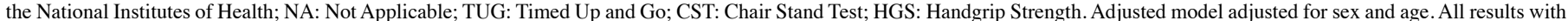
a p-value $<0.05$ are considered significant and are given in bold; *Balance tests were dichotomized into unable to maintain for ten seconds $(0)$ and able to maintain for ten seconds ( 1$)$. 
PHYSICAL PERFORMANCE AND SARCOPENIA

Table 4

Diagnostic accuracy of physical performance measures according to the applied definitions of sarcopenia

\begin{tabular}{|c|c|c|c|c|c|c|}
\hline & & Baumgartner & Janssen & EWGSOP & IWGS & FNIH \\
\hline \multirow[t]{3}{*}{ Side-by-side, eyes open* } & Sensitivity & NA & NA & NA & NA & 20.0 \\
\hline & Specificity & NA & NA & NA & NA & 97.0 \\
\hline & AUC & NA & NA & NA & NA & 0.59 [0.30-0.87] \\
\hline \multirow[t]{3}{*}{ Tandem, eyes open* } & Sensitivity & 82.1 & NA & 75.8 & 82.1 & NA \\
\hline & Specificity & 42.9 & NA & 42.1 & 42.9 & NA \\
\hline & AUC & $0.63[0.52-0.73]$ & NA & $0.59[0.48-0.70]$ & $0.63[0.52-0.73]$ & NA \\
\hline \multirow[t]{3}{*}{ Side-by-side, eyes closed* } & Sensitivity & NA & NA & 36.4 & NA & NA \\
\hline & Specificity & NA & NA & 88.6 & NA & NA \\
\hline & AUC & NA & NA & $0.63[0.51-0.74]$ & NA & NA \\
\hline \multirow[t]{3}{*}{ CST, dichotomized } & Sensitivity & NA & NA & 81.8 & 85.7 & NA \\
\hline & Specificity & NA & NA & 34.3 & 34.5 & NA \\
\hline & AUC & NA & NA & $0.42[0.31-0.53]$ & $0.40[0.29-0.51]$ & NA \\
\hline \multirow[t]{3}{*}{ HGS, dichotomized } & Sensitivity & 50.0 & NA & NA & 53.6 & NA \\
\hline & Specificity & 75.0 & NA & NA & 75.9 & NA \\
\hline & AUC & $0.63[0.50-0.75]$ & NA & NA & $0.65[0.53-0.77]$ & NA \\
\hline
\end{tabular}

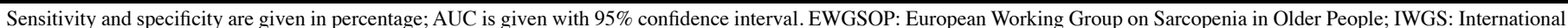

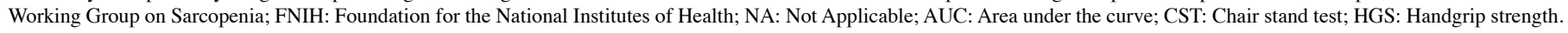

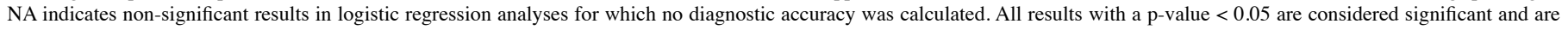
given in bold. *Balance tests were dichotomized into unable to maintain for ten seconds (0) and able to maintain for ten seconds (1).

diagnostic accuracy of physical performance measures to identify older individuals with sarcopenia because physical performance could also be inflicted by disease-specific mechanisms.

To the best of our knowledge, this is the first study outlining the association between various objective physical performance measures and several commonly used definitions of sarcopenia in a clinically relevant population of geriatric outpatients. Validated physical performance measures were used. Furthermore, the population is heterogeneous and no exclusion criteria were used which makes it a good representation of the actual older population visiting outpatient clinics. A limitation of this study could be that only BIA and not Dual Energy X-ray Absorptiometry (DEXA) was used to determine muscle mass parameters of sarcopenia. However, BIA and DEXA showed high agreement in a population of community-dwelling individuals (19).

Single physical performance measures could not identify older individuals with sarcopenia, according to five different definitions. Therefore, no easy and fast method to identify individuals with sarcopenia can be recommended. Future research should focus on developing and validating screening tools so that individuals with a high probability of having sarcopenia can be identified. Individuals who are considered to be at risk of sarcopenia should be referred to diagnose sarcopenia using the diagnostic criteria that are used in the definitions of sarcopenia.
Acknowledgements: We thank M. Stijntjes and J.H. Pasma for their contribution to the study.

Funding: This study was supported by the Dutch Technology Foundation STW, which is part of the Netherlands Organization for Scientific Research (NWO) and which is partly funded by the Ministry of Economic Affairs. Furthermore, this study was supported by the seventh framework program MYOAGE (HEALTH-2007-2.4.5-10) and 050-060-810 Netherlands Consortium for Healthy Aging (NCHA). The funders had no role in study design, data collection and analysis, decision to publish, or preparation of the manuscript.

Statement of authorship: All authors have made substantial contributions to all of the following: 1) conception and design of the study, acquisition of data or analysis and interpretation of data; 2) drafting the article or revising it critically for important intellectual content; 3 ) final approval of the version to be submitted.

Conflict of Interest: S.M.L.M. Looijaard: none to declare. S.J. Oudbier: none to declare. E.M. Reijnierse: none to declare. G.J. Blauw: none to declare. C.G.M. Meskers: none to declare. A.B. Maier: none to declare.

Open Access: This article is distributed under the terms of the Creative Commons Attribution 4.0 International License (http://creativecommons.org/licenses/by/4.0/), which permits use, duplication, adaptation, distribution and reproduction in any medium or format, as long as you give appropriate credit to the original author(s) and the source, provide a link to the Creative Commons license and indicate if changes were made. 


\section{THE JOURNAL OF FRAILTY \& AGING}

\section{References}

1. Reijnierse EM, Trappenburg MC, Leter MJ, et al. The Impact of Different Diagnostic Criteria on the Prevalence of Sarcopenia in Healthy Elderly Participants and Geriatric Outpatients. Gerontology. 2015;61(6):491-6.

2. Beaudart C, Reginster JY, Petermans J, et al. Quality of life and physical components linked to sarcopenia: The SarcoPhAge study. Exp Gerontol. 2015;69:103-10.

3. Landi F, Liperoti R, Russo A, et al. Sarcopenia as a risk factor for falls in elderly individuals: results from the ilSIRENTE study. Clin Nutr. 2012;31(5):652-8.

4. Landi F, Cruz-Jentoft AJ, Liperoti R, et al. Sarcopenia and mortality risk in frail older persons aged 80 years and older: results from ilSIRENTE study. Age Ageing. 2013;42(2):203-9.

5. Baumgartner RN, Koehler KM, Gallagher D, et al. Epidemiology of sarcopenia among the elderly in New Mexico. Am J Epidemiol. 1998;147(8):755-63.

6. Janssen I, Heymsfield SB, Ross R. Low relative skeletal muscle mass (sarcopenia) in older persons is associated with functional impairment and physical disability. J Am Geriatr Soc. 2002;50(5):889-96.

7. Cruz-Jentoft AJ, Baeyens JP, Bauer JM, et al. Sarcopenia: European consensus on definition and diagnosis: Report of the European Working Group on Sarcopenia in Older People. Age Ageing. 2010;39(4):412-23.

8. Studenski SA, Peters KW, Alley DE, et al. The FNIH sarcopenia project: rationale, study description, conference recommendations, and final estimates. J Gerontol A Biol Sci Med Sci. 2014;69(5):547-58.

9. Fielding RA, Vellas B, Evans WJ, et al. Sarcopenia: an undiagnosed condition in older adults. Current consensus definition: prevalence, etiology, and consequences. International working group on sarcopenia. J Am Med Dir Assoc. 2011;12(4):249-56.

10. Janssen I, Baumgartner RN, Ross R, Rosenberg IH, Roubenoff R. Skeletal muscle cutpoints associated with elevated physical disability risk in older men and women. Am J Epidemiol. 2004;159(4):413-21.

11. Reijnierse EM, de van der Schueren MAE, Trappenburg MC, Doves M, Meskers CGM, Maier AB. Lack of knowledge and availability of diagnostic equipment could hinder the diagnosis of sarcopenia and its management. PloS one. 2017;12(10):e0185837.

12. Martinez BP, Gomes IB, Oliveira CS, et al. Accuracy of the Timed Up and Go test for predicting sarcopenia in elderly hospitalized patients. Clinics. 2015;70(5):369-72.

13. Bijlsma AY, Meskers CG, van den Eshof N, et al. Diagnostic criteria for sarcopenia and physical performance. Age. 2014;36(1):275-85.

14. Pinheiro PA, Carneiro JA, Coqueiro RS, Pereira R, Fernandes MH. "Chair Stand Test" as Simple Tool for Sarcopenia Screening in Elderly Women. J Nutr Health Aging. 2016;20(1):56-9.

15. Gray M, Glenn JM, Binns A. Predicting sarcopenia from functional measures among community-dwelling older adults. Age. 2016;38(1):22.

16. Folstein MF, Folstein SE, McHugh PR. "Mini-mental state". A practical method for grading the cognitive state of patients for the clinician. J Psychiatr Res. 1975;12(3):189-98
17. Guralnik JM, Simonsick EM, Ferrucci L, et al. A short physical performance battery assessing lower extremity function: association with self-reported disability and prediction of mortality and nursing home admission. J Gerontol. 1994;49(2):85-94.

18. Reijnierse EM, de Jong N, Trappenburg MC, et al. Assessment of maximal handgrip strength: how many attempts are needed? J Cachexia Sarcopenia Muscle. 2017;8(3):466-74.

19. Ling $\mathrm{CH}$, de Craen AJ, Slagboom PE, et al. Accuracy of direct segmental multifrequency bioimpedance analysis in the assessment of total body and segmental body composition in middle-aged adult population. Clin Nutr. 2011;30(5):610-5.

20. Nishimura T, Arima K, Okabe T, et al. Usefulness of chair stand time as a surrogate of gait speed in diagnosing sarcopenia. Geriatr Gerontol Int. 2017;17(4):659-61.

21. Lauretani F, Russo CR, Bandinelli S, et al. Age-associated changes in skeletal muscles and their effect on mobility: an operational diagnosis of sarcopenia. J Appl Physiol. 2003;95(5):1851-60.

22. Freiberger E, de Vreede P, Schoene D, et al. Performance-based physical function in older community-dwelling persons: a systematic review of instruments. Age Ageing 2012;41(6):712-21.

23. Stevens PJ, Syddall HE, Patel HP, Martin HJ, Cooper C, Aihie Sayer A. Is grip strength a good marker of physical performance among community-dwelling older people? J Nutr Health Aging. 2012;16(9):769-74.

24. Sanchez-Rodriguez D, Marco E, Miralles R, et al. Does gait speed contribute to sarcopenia case-finding in a postacute rehabilitation setting? Arch Gerontol Geriatr. 2015;61(2):176-81.

25. Stoever K, Heber A, Eichberg S, Brixius K. Sarcopenia and Predictors of Skeletal Muscle Mass in Elderly Men With and Without Obesity. Gerontol Geriatr Med. 2017;3:2333721417713637.

26. Verlaan S, Maier AB, Bauer JM, et al. Sufficient levels of 25-hydroxyvitamin D and protein intake required to increase muscle mass in sarcopenic older adults - The PROVIDE study. Clin Nutr. 2018;37(2):551-7.

27. Beaudart C, Dawson A, Shaw SC, et al. Nutrition and physical activity in the prevention and treatment of sarcopenia: systematic review. Osteoporosis Int 2017;28(6):1817-33.

28. Thibault R, Genton L, Pichard C. Body composition: why, when and for who? Clin Nutr. 2012;31(4):435-47.

29. Malmstrom TK, Miller DK, Simonsick EM, Ferrucci L, Morley JE. SARC-F: a symptom score to predict persons with sarcopenia at risk for poor functional outcomes. J Cachexia, Sarcopenia Muscle. 2016;7(1):28-36.

30. Rolland Y, Dupuy C, Abellan Van Kan G, et al. Sarcopenia Screened by the SARC-F Questionnaire and Physical Performances of Elderly Women: A Cross-Sectiona Study. J Am Med Dir Assoc. 2017;18(10):848-52. 